Noting that the invasive strategy was safe and reduced the duration of hospital stay, the authors suggest that it may be more appropriate than conservative treatment.

Original article Fernandez-Avilés F et al. (2004) Routine invasive strategy within 24 hours of thrombolysis versus ischaemia-guided conservative approach for acute myocardial infarction with ST-segment elevation (GRACIA-1): a randomised controlled trial. Lancet 364: 1045-1053

\section{Reduced risk of stroke with candesartan}

Stroke is the major cardiovascular complication of hypertension in older patients. A new study has shown a reduced risk in selected patients receiving candesartan, an angiotensin II type 1 receptor blocker.

In the Study on Cognition and Prognosis in the Elderly (SCOPE), patients were randomly assigned to receive candesartan or placebo, plus open-label antihypertensive therapy as necessary. Since isolated systolic hypertension (ISH) is the predominant form of hypertension in older patients, Papademetriou et al. carried out a predefined subgroup analysis of 1,518 patients with ISH (defined as systolic blood pressure $\geq 160 \mathrm{mmHg}$ and diastolic blood pressure $<90 \mathrm{mmHg}$ ). Bloodpressure lowering and cardiovascular events were compared between the candesartan and control groups.

After a mean follow-up of 3.6 years, blood pressure reduction was slightly greater $(2.0 / 1.2 \mathrm{mmHg})$ in patients treated with candesartan $(n=754)$, than in the control group $(n=764)$, but this difference was not statistically different. Adjusting for baseline risk, however, there was a $42 \%$ relative risk reduction in stroke in the candesartan group (20 vs 35 fatal/nonfatal strokes; $P=0.049$ ).

The authors propose that the observed reduction in stroke risk might have been partly due to the slight improvement in blood pressure control afforded by candesartan treatment. They also suggest that the vascular protective effects of this class of drugs may be responsible for the benefit.

Original article Papademetriou V et al. (2004) Stroke prevention with the angiotensin II type 1-receptor blocker candesartan in elderly patients with isolated systolic hypertension. J Am Coll Cardiol 44: 1175-1180

\section{Role of the native heart following transplant}

Hypertension frequently develops following organ transplantation, partly as a consequence of ciclosporin treatment. Since hypertension is more common in heart transplant patients than in liver or lung transplant recipients, it is possible that removal of the recipient's heart contributes to the problem. In orthotopic cardiac transplant (OCT), the native heart is replaced with the denervated donor heart. In heterotopic heart transplant (HHT), a less common procedure, the recipient heart and its innervation are left in situ. Taegtmeyer et al. hypothesized that the incidence of hypertension in HHT patients would, therefore, be lower than in OCT patients.

The authors examined retrospective data from 271 patients, of whom 233 had undergone OCT and 38 had undergone HHT. At 3 months post-transplantation, systolic blood pressure was significantly lower in the HHT group than in the OCT group, despite greater use of antihypertensive drugs in the OCT patients. This difference in systolic blood pressure persisted throughout the 5year period studied. Multivariate analysis revealed that, compared with OCT, HHT was independently associated with lower systolic blood pressure.

The study supports the idea that the native heart has a role in blood pressure homeostasis following $\mathrm{HHT}$, and suggests that its removal is involved in the development of hypertension after OCT.

Original article Taegtmeyer AB et al. (2004) Reduced incidence of hypertension after heterotopic cardiac transplantation compared with orthotopic cardiac transplantation. J Am Coll Cardiol 44: 1254-1260

\section{Stenting versus surgery in carotid artery stenosis}

Severe carotid artery stenosis is routinely treated by endarterectomy to prevent stroke. Although this strategy tends to be superior to medical management, the surgery carries a high risk in some patients. Carotid angioplasty is a less invasive option but has been hampered by stent compression and embolization of plaque debris. Recently, crush-resistant stents and 